\title{
Left ventricular dysfunction in heart failure with preserved ejection fraction-molecular mechanisms and impact on right ventricular function
}

\author{
Frank R. Heinzel ${ }^{1,2,3}$, Niklas Hegemann ${ }^{1,4}$, Felix Hohendanner ${ }^{1,2,3}$, Uwe Primessnig ${ }^{1,2,3}$, Jana Grune ${ }^{2,3,4}$, \\ Florian Blaschke ${ }^{1,2,3}$, Rudolf A. de Boer ${ }^{5}$, Burkert Pieske ${ }^{1,2,3,6}$, Gabriele G. Schiattarella ${ }^{7}$, \\ Wolfgang M. Kuebler ${ }^{2,3,4}$
}

${ }^{1}$ Department of Internal Medicine and Cardiology, Charité-Universitätsmedizin Berlin, Campus Virchow-Klinikum, Berlin, Germany; ${ }^{2}$ DZHK (German Centre for Cardiovascular Research), Partner Site Berlin, Germany; ${ }^{3}$ Berlin Institute of Health (BIH), Berlin, Germany; ${ }^{4}$ Institute of Physiology, Charité-Universitätsmedizin Berlin, Berlin, Germany; ${ }^{5}$ Department of Cardiology, Groningen, University Medical Center Groningen, University of Groningen, The Netherlands; ${ }^{6}$ Department of Internal Medicine and Cardiology, German Heart Center, Berlin, Germany; ${ }^{7}$ Department of Internal Medicine-Cardiology, UT Southwestern Medical Center, Dallas, TX, USA

Contributions: (I) Conception and design: None; (II) Administrative support: None; (III) Provision of study materials or patients: None; (IV) Collection and assembly of data: None; (V) Data analysis and interpretation: None; (VI) Manuscript writing: All authors; (VII) Final approval of manuscript: All authors.

Correspondence to: Prof. Frank R. Heinzel, MD, PhD. Department of Internal Medicine and Cardiology, Charité-Universitätsmedizin Berlin, Campus Virchow-Klinikum, Augustenburgerplatz 1, 13353 Berlin, Germany. Email: frank.heinzel@charite.de.

\begin{abstract}
The current classification of heart failure (HF) based on left ventricular (LV) ejection fraction (EF) identifies a large group of patients with preserved ejection fraction (HFpEF) with significant morbidity and mortality but without prognostic benefit from current HF therapy. Co-morbidities and conditions such as arterial hypertension, diabetes mellitus, chronic kidney disease, adiposity and aging shape the clinical phenotype and contribute to mortality. LV diastolic dysfunction and LV structural remodeling are hallmarks of HFpEF, and are linked to remodeling of the cardiomyocyte and extracellular matrix. Pulmonary hypertension (PH) and right ventricular dysfunction (RVD) are particularly common in HFpEF, and mortality is up to 10 -fold higher in HFpEF patients with vs. without RV dysfunction. Here, we review alterations in cardiomyocyte function (i.e., ion homeostasis, sarcomere function and cellular metabolism) associated with diastolic dysfunction and summarize the main underlying cellular pathways. The contribution and interaction of systemic and regional upstream signaling such as chronic inflammation, neurohumoral activation, and NO-cGMP-related pathways are outlined in detail, and their diagnostic and therapeutic potential is discussed in the context of preclinical and clinical studies. In addition, we summarize prevalence and pathomechanisms of RV dysfunction in the context of HFpEF and discuss mechanisms connecting LV and RV dysfunction in HFpEF. Dissecting the molecular mechanisms of LV and RV dysfunction in HFpEF may provide a basis for an improved classification of $\mathrm{HFpEF}$ and for therapeutic approaches tailored to the molecular phenotype.
\end{abstract}

Keywords: Heart failure (HF); cardiomyocytes; metabolism; calcium; sodium; right ventricle; pulmonary hypertension $(\mathrm{PH})$

Submitted May 02, 2020. Accepted for publication Aug 27, 2020.

doi: $10.21037 / \mathrm{cdt}-20-477$

View this article at: http://dx.doi.org/10.21037/cdt-20-477 


\section{Heart failure with preserved ejection fraction (HFpEF)—clinical and cellular characteristics}

\section{Introduction}

A 71 years old mildly overweight woman is transferred to the hospital with worsening dyspnea on exertion, unproductive cough and mild ankle edema for a few weeks. Once the diagnosis of heart failure (HF) is established, she faces a 1 in 6 chance to die within 2 years despite optimal current therapy (1). HF is the leading cause for hospital admissions, and more than 26 million people worldwide are affected (2). HF is diagnosed in a large variety of patients with different medical backgrounds. Currently, diagnosis is based on clinical signs and symptoms, and imaging (e.g., echocardiography). HF patients are classified based on left ventricular $(\mathrm{LV})$ ejection fraction $(\mathrm{EF})$ as patients with preserved (HFpEF, LV EF $\geq 50 \%$ ), reduced (HFrEF, LV EF $<40 \%$ ) or mid-range $\mathrm{EF}$ as basis for clinical management. Inhibition of neurohumoral activation with ACEinhibitors, angiotensin-receptor blockers or betablockers, and enhancing the availability of natriuretic peptides (NP) with neprilysin inhibitors (sacubitril, in combination with valsartan) are effective treatments in HFrEF but failed to improve prognosis in HFpEF (3-5) (see also "neurohumoral activation" section for discussion). It is commonly argued that HFpEF represents a more heterogeneous disease as suggested by (I) the variety of clinical disease conditions associated with HFpEF (6), (II) very different long-term prognosis if HFpEF patients are classified based on the severity of their predominant clinical features (7), and (III) identification of subgroups of HFpEF patients that respond to medical HF therapy in pooled analyses from randomized controlled trials (RCTs) and observational trials $(5,8)$. Some but not all studies suggest an increased number of comorbidities in HFpEF vs. HFrEF $(9,10)$. HFpEF patients are older and more likely female (11). Renal failure seems equally common in all HF patients, however, other so-called "non-cardiac" co-morbidities such as chronic obstructive pulmonary disease (COPD), diabetes, obesity and arterial hypertension are more prevalent in $\operatorname{HFpEF}(10,11)$.

The cardiac phenotype of HFpEF is characterized by increased LV filling pressure, a reduced LV stroke volume (SV) (at rest or during exercise) and blunted chronotropic response in some patients. Cardiac output (CO) greatly depends on myocardial pump function as well as the elastic properties of the subsequent arterial vasculature and venous blood flow returning to the heart. Their contribution may be of particular importance for right heart function in $\mathrm{HF}$ as discussed below (see "RVD in HFpEF"). Ventricular aortic coupling is reviewed in detail by Bernardo et al. in this issue of the Journal. Here, we will focus on molecular and cellular mechanisms of HFpEF at the level of the myocardium.

In the canonical view of $\mathrm{HF}(\mathrm{rEF})$ pathophysiology, neurohumoral activation and maladaptive cardiac remodeling are triggered by a reduction in myocardial contractile force that results from the replacement of functional cardiomyocytes by fibrotic scar tissue [e.g., following necrosis, apoptosis or both (12-15)]. While fibrosis is also a hallmark of remodeling in HFpEF (see "fibrosis/extracellular matrix" section), excessive cardiomyocyte death is not typical (16), even though an increased rate of apoptosis is observed in specific cardiomyopathies $(17,18)$. Disease progression in HF is paralleled by an increased mechanical load on the cardiac myocytes, either by increased preload despite reduced $\mathrm{SV}$ or by increased afterload with the progression of comorbidities. Cardiac remodeling in HF is almost invariably associated with cardiomyocyte hypertrophy, which may be even more pronounced in HFpEF vs. HFrEF (19). In $\mathrm{HFpEF}$, cardiomyocytes contribute to diastolic dysfunction by increased passive stiffness (see "the role of titin in impaired cardiomyocyte relaxation" and "inflammatorydriven pathogenic pathways in HFpEF" sections). However, recent evidence indicates that systemic inflammation and metabolic dysregulation also impair excitation-contraction coupling (ECC) and thus active cardiomyocyte relaxation as discussed in "active cardiomyocyte relaxation" section. Hypertrophic cardiomyopathy (HCM), a genetic disorder of the myofilaments, is often associated with diastolic dysfunction, and-even in the absence of outflow tract obstruction-may lead to HFpEF (20). While implications of alterations in myofilament properties are discussed in "myofilament properties" section, we refer to more detailed recent reviews on the pathophysiology and presentation of HCM (20-22).

\section{Increased myocardial passive stiffness}

Increased myocardial passive stiffness is a hallmark feature of HFpEF. Two major components are relevant for increased passive stiffness: altered extracellular matrix composition and impaired cardiomyocyte relaxation.

\section{Fibrosis/extracellular matrix}

As extensively reviewed before (13,23-25), the amount of collagen, the abundance of collagen type 1 and collagen 
cross-linking contribute to increased myocardial stiffness and diastolic dysfunction. In MRI studies, impaired diastolic function correlated with myocardial fibrosis (26), which is predominantly localized perivascularly and diffusely in the interstitial space $(26,27)$ and is considered a hallmark feature of HFpEF (28). Fibrosis has also been related to inflammatory triggers (29). Consistently, myocardial inflammation has also been linked to increased collagen type 1 and enhanced collagen cross-linking in biopsies from HFpEF patients (30). Extracellular matrix is also influenced by other proteins that accumulate in the setting of comorbidities frequently associated with HFpEF. For instance, advanced glycosylation end-products (AGEs) are found in particular in patients with diabetes (31) and have been associated with myocardial inflam $\neg$ mation and HFpEF (32), although their role for impaired relaxation is debated (31). Interestingly, extracellular matrix stiffness has been shown to directly affect cardiomyocyte function and $\mathrm{Ca}^{2+}$ transients (CaTs) in a HFpEF model (33).

\section{The role of titin in impaired cardiomyocyte relaxation}

Titin exists in two main isoforms that contribute to myocardial stiffness, the $\mathrm{N} 2 \mathrm{~B}$ and the $\mathrm{N} 2 \mathrm{Ba}$ isoform springs. As these isoforms show different compliances titinisoform switch, i.e., an altered N2B/N2BA ratio, is a well understood mechanism contributing to increased diastolic stiffness in a variety of cardiomyopathies (34). It is thus not surprising that isoform switch was identified as a main driver of HFpEF development (7). In addition, titin compliance is influenced by its phosphorylation state, tightly related to local inflammation and oxidative stress (35). It has been suggested that titin and cardiomyocyte related alterations are more important during early as compared to late stages of HFpEF (36). Interestingly, arterial hypertension with LV hypertrophy but without HF was not associated with alterations in titin or myocardial stiffness (24). The pathophysiology of titin has been extensively reviewed elsewhere (36-38). We address titin-based therapeutic approaches in "cGMP—signaling" section.

\section{Active cardiomyocyte relaxation}

Whereas passive mechanical properties of cardiomyocytes are evaluated in skinned myofibers obtained from animals and from human myocardial biopsies ("the role of titin in impaired cardiomyocyte relaxation" section), examination of active contraction and relaxation during ECC requires access to intact and functional myocardium and isolated cardiomyocytes (quantitative measurements of $\mathrm{Ca}^{2+}$ and $\mathrm{Na}^{+}$). Due to limited access to such samples from HFpEF patients, current data on ECC is mainly based on animal models. Advantages and limitations of currently available models have been comprehensively discussed recently (39).

\section{Altered cytosolic $\mathrm{Ca}^{2+}$ - and $\mathrm{Na}^{+}$-signaling}

Cardiomyocyte contraction and relaxation is governed by the shape of the cytosolic CaT activating the myofilaments. $\mathrm{Ca}^{2+}$ release into the cytosol from the sarcoplasmic reticulum (SR) through ryanodine receptors (RyR2) is triggered by $\mathrm{Ca}^{2+}$ influx through L-type $\mathrm{Ca}^{2+}$ channels (LTCC) during the action potential $\left(\mathrm{Ca}^{2+}\right.$-induced $\mathrm{Ca}^{2+}$ release) and is highly controlled within intracellular microdomains (dyads). Cytosolic $\mathrm{Ca}^{2+}$ removal is achieved mainly by the ATPdependent SR Ca ${ }^{2+}$ pump (SERCA) and to a lesser extent by extrusion via the sarcolemmal $\mathrm{Na}^{+} / \mathrm{Ca}^{2+}$ exchanger (NCX) balancing the influx of $\mathrm{Ca}^{2+}$ via LTCC during the cardiac cycle. Cardiomyocytes from end-stage HFrEF are characterized by increased diastolic $\left[\mathrm{Ca}^{2+}\right]_{i}$, reduced peak $\left[\mathrm{Ca}^{2+}\right]_{\mathrm{i}}$, a slower cytosolic $\mathrm{Ca}^{2+}$ removal and reuptake into the SR and a lower SR $\left[\mathrm{Ca}^{2+}\right]$. Most consistently related is a reduced activity of SERCA and increased SR $\mathrm{Ca}^{2+}$ leak through RyR2. In recent years, with the development of animal models more closely mimicking the co-incidence of relevant triggering co-morbidities in vivo, distinct and more diverse cardiomyocyte phenotypes have been described in HFpEF. As summarized in Table 1 alterations in cytosolic $\left[\mathrm{Ca}^{2+}\right]$ have been observed in a variety of models in isolated cardiomyocytes ex vivo, indicating an adaptation of cardiomyocyte ECC in HFpEF even in the absence of the systemic metabolic and hemodynamic challenges present in vivo. Levels of diastolic $\left[\mathrm{Ca}^{2+}\right]$ are often increased which not only reflects impaired relaxation but also influences the effects of $\mathrm{Ca}^{2+}$ release following depolarization (50). Cytosolic $\left[\mathrm{Ca}^{2+}\right]$ decay is often prolonged, suggesting a contribution of prolonged active relaxation to diastolic dysfunction. In support, a close correlation between the $\left[\mathrm{Ca}^{2+}\right]$ decay constant in isolated cardiomyocytes and the isovolumetric relaxation time in vivo has been documented in a cardiorenal HFpEF model (47). SR Ca ${ }^{2+}$ leak may be a sign of advanced stages of HFpEF (47). In contrast to end-stage $\mathrm{HFrEF}$, the CaT amplitude for $\mathrm{SR}\left[\mathrm{Ca}^{2+}\right]$, where determined $\}$ is not generally reduced and maybe even increased thus resembling the findings in "compensated" LV hypertrophy (51). Indeed, in a novel hypertrophic heart rat model without hypertension, diastolic dysfunction and congestion were associated 
Table 1 Altered cardiomyocyte $\mathrm{Ca}^{2+}$ homeostasis in different preclinical models of $\mathrm{HFpEF}$

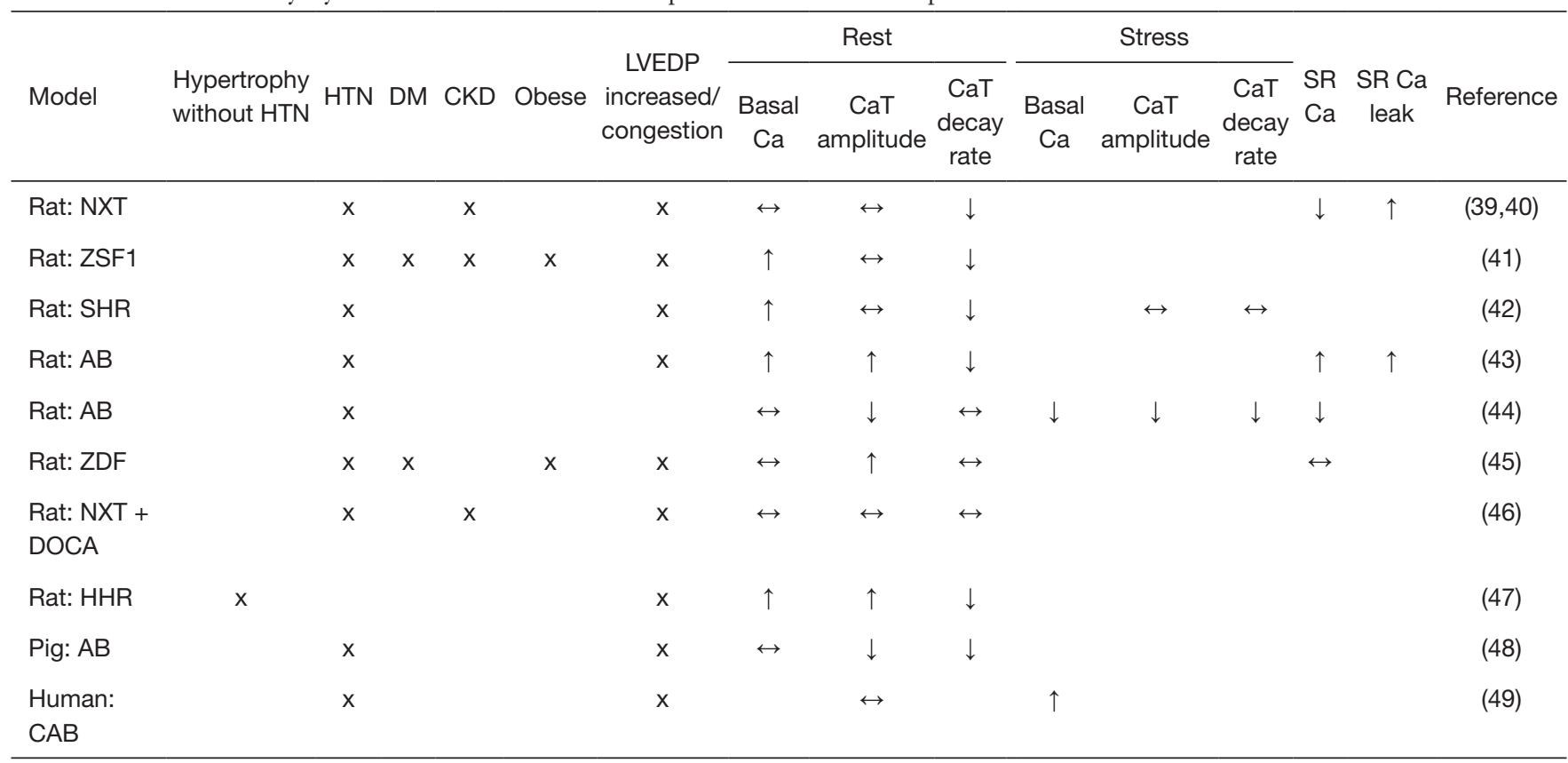

$\mathrm{x}$, clinical condition; $\leftrightarrow$, unchanged vs. control; $\uparrow$, increased vs. control; $\downarrow$, decreased $v$ s. control. HFpEF, heart failure with preserved ejection fraction; HTN, hypertension; DM, diabetes mellitus; CKD, chronic kidney disease; LVEDP, left ventricular end-diastolic pressure; CaT, Ca ${ }^{2+}$ transient; SR, sarcoplasmic reticulum; NXT, subtotal nephrectomy; ZDF, Zucker diabetic fatty rat; SHR, spontaneously hypertensive rat; ZSF1, ZDF crossed with SHR; AB, aortic banding; HHR, hypertrophic heart rat; DOCA, chronic deoxycorticosterone acetate treatment; $\mathrm{CAB}$, coronary artery bypass with HFpEF.

with increased LTCC influx and CaT amplitudes which may indicate a compensatory increase in inotropy in response to fibrotic hypertrophic remodeling (40). On the other hand, modeling of human myocardium suggests that a concentrically hypertrophied ventricular wall can maintain a preserved EF even despite reduced sarcomere shortening at the cardiomyocyte level (41) as implicated by reduced CaT amplitudes observed in some studies (Table 1).

It must be noted that only a minority of studies evaluated the functional response of cardiomyocytes from HFpEF models on physiological stressors (e.g., pacing frequency, adrenergic stimulation). Thus, more data is needed to elucidate the heterogeneity of the cardiomyocytes' functional response to exercise-related stimuli in HFpEF.

As opposed to end-stage HFrEF, a uniform pattern of expression of $\mathrm{Ca}^{2+}$ handling proteins has not emerged in HFpEF, but different modalities of post-translational regulation (e.g., oxidation, glycation, $S$-nitrosylation, phosphorylation) may contribute to altered $\mathrm{Ca}^{2+}$ trafficking, probably depending on HFpEF etiology $(42,43)$. Also, increased cytosolic $\mathrm{Ca}^{2+}$ buffering slows $\mathrm{Ca}^{2+}$ trafficking and may decelerate $\mathrm{Ca}^{2+}$ decay and increase diastolic $\mathrm{Ca}^{2+}$ especially at higher heart rates $(44,50)$.

Intracellular $\left[\mathrm{Ca}^{2+}\right]$ is closely linked to $\left[\mathrm{Na}^{+}\right]$via the NCX which contributes to cytosolic $\mathrm{Ca}^{2+}$ removal even more in larger species and humans as compared to rodents (45). Increased $\left[\mathrm{Na}^{+}\right]$near the NCX can strongly influence the balance of transsarcolemmal $\mathrm{Ca}^{2+}$ fluxes and may thus contribute to altered cardiomyocyte relaxation in HFrEF $(46,48)$. Potential mechanisms leading to intracellular $\mathrm{Na}^{+}$ accumulation in cardiomyocytes include late $\mathrm{Na}^{+}$influx via fast (Nav1.5) $\mathrm{Na}^{+}$channels (INa,late), increased activity of the $\mathrm{Na}^{+} / \mathrm{H}^{+}$exchanger (NHE1), reduced activity of the ATP-driven $\mathrm{Na}^{+} / \mathrm{K}^{+}$-pump (NKA), and influx via the sodium glucose co-transporter (SGLT1), the $\mathrm{Na}^{+} / \mathrm{K}^{+} /$ $\mathrm{Cl}^{-}$co-transporter (NKCC1) or via less selective cation channels (e.g., TRP-channels). NCX undergoes complex mode shifts (forward/reverse) during the same cardiac cycle and may itself be a relevant source of $\mathrm{Na}^{+}$influx into cardiomyocytes (49). However, the role of $\left[\mathrm{Na}^{+}\right]_{\mathrm{i}}$ as a driver for diastolic dysfunction and the relevance of any of the mentioned mechanisms of $\mathrm{Na}^{+}$accumulation in cardiomyocytes in HFpEF is still under debate $(47,52,53)$. 
While experimental data, also in human myocardium, support the concept to treat diastolic dysfunction by inhibition of INa,late in a variety of cardiac disease models (54-57), the INa,late inhibitor ranolazine failed to improve diastolic function acutely and after $14 \mathrm{~d}$ in a small cohort of patients with HFpEF in a clinical phase IIa proof-of-concept study (RALI-DHF) (58). In a more recent study, muscle strips from the LV anterior wall (epicardial biopsies) of patients with hypertensive heart disease and HFpEF showed a prolonged relaxation time and a rate-induced increase in diastolic $\left[\mathrm{Ca}^{2+}\right]$. However, no frequency-dependent increase in cytosolic $\left[\mathrm{Na}^{+}\right]$or altered expression of $\mathrm{Na}^{+}-$/ $\mathrm{Ca}^{2+}$ handling proteins were detected, and neither the acute application of ranolazine, amiloride (inhibiting NHE1), or furosemide (targeting NKCC1) improved cardiomyocyte relaxation (59). These results have been interpreted to argue against a role of increased cardiomyocyte $\left[\mathrm{Na}^{+}\right]_{\mathrm{i}}$ in HFpEF $(16,59)$. However, the complex regulation of sarcolemmal $\left[\mathrm{Na}^{+}\right]$fluxes may not be reflected in the bulk cytosol $(60,61)$, and the contrasting findings in different models warrant a more quantitative assessment of intracellular $\left[\mathrm{Na}^{+}\right]$ dynamics and the evaluation of long-term adaptation of the cardiomyocyte to $\left[\mathrm{Na}^{+}\right]$influx modulation in different etiologies of HFpEF.

\section{Myofilament properties}

HCM is often associated with global hypercontractility but also diastolic dysfunction as characterized e.g., by increased LV end-diastolic pressure or LA remodeling, which may result in overt HFpEF (20). Depending on the underlying gene mutation, different micro- and macroscopic myocardial HCM phenotypes have been characterized (21). While myocardial passive stiffness is increased (by interstitial fibrosis and increased wall thickness), these effects are likely sequelae of dysfunctional sarcomeres. At the myofilaments, specific mutations have been associated with myofilament disarray with impaired $\mathrm{Ca}^{2+}$ cycling, an increased energy cost of contraction and/or pathologically increased myosin-actin binding during relaxation and even in the resting state (21). Based on the latter, a novel therapeutic approach currently in clinical trials investigates the effects of a first-of-its class myosin-ATPase inhibitor, mavacamten, in patients with symptomatic HCM or hypertrophic obstructive cardiomyopathy (HOCM) (62-64). First results of recent clinical phase 2 studies suggest good tolerability and improvement in some parameters of diastolic dysfunction $(62,64)$.

While myofilament properties have been extensively studied in hypertrophic and dilated cardiomyopathy (65), the contribution of altered $\mathrm{Ca}^{2+}$ sensitivity and cross-bridge cycling to contractile dysfunction in other, more common etiologies of HFpEF is less well understood. Binding of $\mathrm{Ca}^{2+}$ to the troponin complex of the thin filament depends on the dissociation constant of troponin $\mathrm{C}(\mathrm{TnC})$. Adrenergic, PKA-dependent phosphorylation of troponin I (TnI, at Ser23/24) and cardiac myosin-binding protein C (cMBP-C) can reduce myofilament $\mathrm{Ca}^{2+}$ sensitivity by an altered $\mathrm{TnC}-\mathrm{TnI}$ interaction and thus enhance relaxation of the myofilaments $(66,67)$. Myosin-ATPase forms the motor of cross-bridge cycling by converting energy into myofilament motion. In HFrEF, an activator of myosinATPase is currently evaluated in clinical trials to improve CO without changing the kinetics of contraction (68), whereas inhibition of a hypercontractile myosin-ATPase in genetic cardiomyopathies has been shown to prevent hypertrophic remodeling (69). The role of myosin-ATPase in HFpEF has not yet been explored.

In one of the few studies on active myofilament function in $\mathrm{HFpEF}$, the expression of the sarcomeric proteins cMyBP-C, cTnI, and cardiac myosin light chain 2 (cMLC2) were found unaltered in old dogs with hypertensioninduced LV hypertrophy and diastolic dysfunction (70). However, phosphorylation of cMyBP-C (-80\% at S282), TnT, TnI, and MLC2 (-70\% at S19) was reduced resulting in increased $\mathrm{Ca}^{2+}$ sensitivity, reduced maximal activated tension, and slowed relaxation. Interestingly, PKA activity was not different but expression of phosphatases (PP1 and PP2a) was increased. Reduced cMYBP-C phosphorylation has been directly linked to diastolic dysfunction (71) and its dysregulation may contribute to $\mathrm{HFpEF}$ in the aging heart (72).

Lovelock et al. also reported increased myofilament sensitization (DOCA HFpEF rat). In this study, glutathionylation rather than phosphorylation of MyBP-C was associated with cardiomyocyte dysfunction (73). Analogously a variety of different post-translational modifications at the level of the myofilament proteins have been associated with diastolic dysfunction, including among others glutathionylation, methylglyoxal modification or oxidation, highlighting the variability and complexity of upstream signaling pathways (74-76).

\section{Metabolic dysregulation}

Metabolic dysregulation-i.e., the clinical alterations of diabetes mellitus, hypercholesterinemia and adiposity-is 


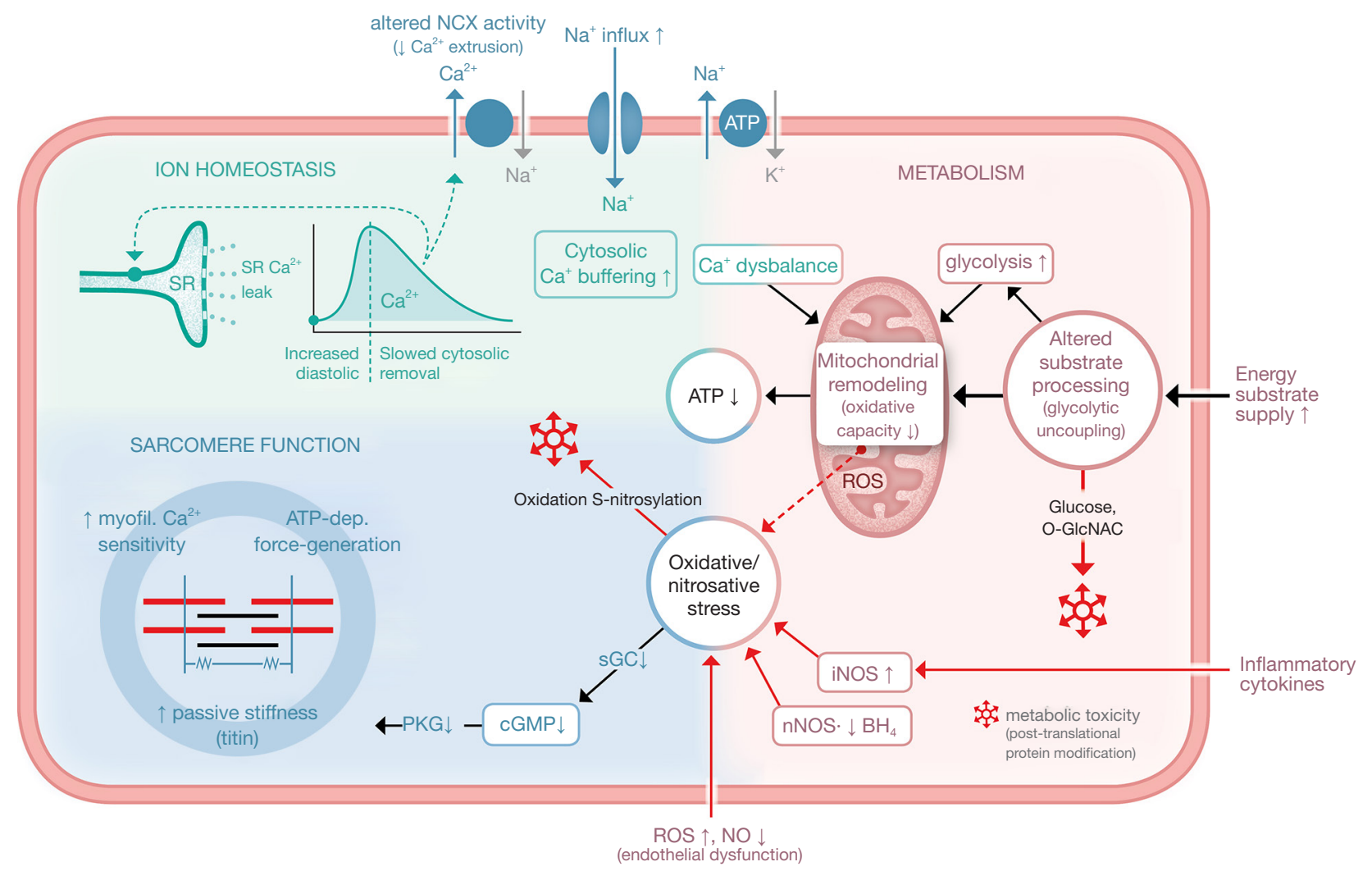

Figure 1 Mechanisms of cardiomyocyte dysfunction in HFpEF. Cardiomyocyte phenotypes include altered ion homeostasis (increased cytosolic $\mathrm{Ca}^{2+}$ and $\mathrm{Na}^{+}$load), increased myofilament $\mathrm{Ca}^{2+}$ sensitivity, slower sarcomere relaxation and increased passive stiffness linked to reduced cGMP-PKG-mediated signaling. Mitochondrial oxidative capacity is reduced due to dysregulated intracellular metabolic processing despite an increased external supply with energy substrates. ROS (from mitochondria and external sources) and reduced NO availability contribute to increased oxidative and nitrosative stress and metabolic toxicity. HFpEF, heart failure with preserved ejection fraction; ROS, reactive oxygen species; $\mathrm{NO}$, nitric oxide.

closely linked to HFpEF and within this population conveys an even worse prognosis $(7,11,77)$. Adiposity, in particular, is an independent risk factor for the manifestation and progression of HFpEF $(78,79)$. A considerable number of patients with HFpEF [24-45\% (11)] suffer from type 2 diabetes mellitus promoting inflammation, increased interstitial fibrosis and collagen stiffening (39). At the cellular level, several aspects of metabolic dysregulation may contribute to HF (80), including an increased supply of the heart with energy substrates ["fuel overload" (81)], accumulation and dysfunctional processing of energy substrates within the myocardium $(82,83)$ (Figure 1), and metabolic imbalance in peripheral tissue due to reduced $\mathrm{CO}$ and consequential reduced oxygen utilization in the peripheral tissue $(84,85)$.
In healthy cardiomyocytes, the vast majority of ATP $(>90 \%)$ is generated in the mitochondria by oxidative phosphorylation of fatty acids (FAs, $60 \%$ ) or glucose $(40 \%)(82)$. Increased glycolysis in combination with unchanged glucose oxidation (i.e., glycolytic uncoupling) was observed early in DOCA HFpEF rats (86). Collectively, the majority of current clinical and experimental studies in diabetic/obese as well as non-diabetics suggest that development of HFpEF (as opposed to HFrEF) is accompanied by increased FA oxidation and reduced glucose oxidation [see (83) for a comprehensive review]. Increased FA uptake is also observed in RV dysfunction [see Agarwal et al. in this issue (doi: 10.21037/cdt-20-404)]. Glucose oxidation is more effective, i.e., it yields more high-energy phosphate bonds per mol $\mathrm{O}_{2}$ than $\mathrm{FA}$, whereas FA yield 
more energy per gram substrate than glucose if oxidation is not limiting (87). Comparison of ZSF-1 (diabetic obese) and DOCA + high salt diet (hypertensive) rat HFpEF models suggests differential regulation of oxidative phosphorylation depending on the HFpEF trigger (88). Also, in a porcine model of HFpEF induced by aortic banding, magnetic resonance spectroscopy imaging indicated a switch from FA towards glucose substrate utilization in the myocardium (89), again highlighting the complexity of HFpEF(-like) phenotypes. As a result of metabolic dysregulation, data from animal experiments suggest a decrease in cardiac (and peripheral) mitochondrial ATP production in hypertrophic hearts with diastolic dysfunction (90). In addition, structural changes associated with a reduced availability of functional mitochondria have been described in HFpEF (91).

In patients with $\mathrm{HFpEF}$ the ratio between cardiac creatine phosphate and adenosine triphosphate is reduced suggesting a reduced energy reserve (92).

In HFrEF mitochondrial remodeling has been linked to an increased mitochondrial production of reactive oxygen species (ROS). It is currently unclear whether mitochondrial ROS production in cardiomyocytes is increased in HFpEF. Another source for ROS is uncoupling of nitric oxide synthase (NOS) by a HFpEF-related reduction of the coenzyme biopterin (93). Increased ROS add to oxidative/ nitrosative stress and may interfere with a variety of intracellular signaling pathways (e.g., via CaMKII) by posttranslational protein modification (Figure 1). ROS formation may be less evident in early HFpEF (94). In light of these findings, therapeutic approaches targeting mitochondria (i.e., to stimulate mitochondrial ATP production or reduce ROS) are currently evaluated (85). It has been proposed but not yet clinically validated that NO-cGMP related signaling may also increase the quantity of functionally active mitochondria thus potentially improving myocardial oxygen consumption (95). Interestingly, mitochondrial dysfunction has also been demonstrated in models of $\mathrm{RV}$ failure as reviewed by Agarwal et al. in this issue (doi: 10.21037/cdt-20-404).

\section{Mitochondrial Ca signaling}

In cardiomyocytes ATP consumption (mainly by the myofilaments) is tightly coupled to ATP production in the mitochondria via $\mathrm{Ca}^{2+}$ fluxes from the cytosol to the mitochondria (96). While disruption of the tight coupling between the cytosolic $\mathrm{Ca}^{2+}$ release units may contribute to impaired myofilament energy supply in HF, high mitochondrial $\mathrm{Ca}^{2+}$ exposure (as in myocardial ischemia with cytosolic $\mathrm{Ca}^{2+}$ overload) induces cardiomyocyte apoptosis (97). In pressure overload-induced LV remodeling this delicate balance may be decisive for the transition from compensated hypertrophy to HF (98). Mitochondrial signaling in $\mathrm{HFpEF}$ is not yet well understood. In a porcine aortic banding model, mitochondria from HFpEF hearts were more susceptible to $\mathrm{Ca}^{2+}$ overload (99). In addition, a recent study showed that increased cytosolic $\mathrm{Ca}^{2+}$ turnover in HFpEF (see "altered cytosolic $\mathrm{Ca}^{2+}$ - and $\mathrm{Na}^{+}$-signaling" section) was associated with increased mitochondrial $\mathrm{Ca}^{2+}$ load leading to reduced oxidative capacity and mitochondrial swelling. Based on these early data, protecting mitochondria from the cytosolic $\mathrm{Ca}^{2+}$ challenge should be evaluated as a therapeutic approach in $\mathrm{HFpEF}$.

\section{HFpEF-alterations in signaling}

\section{Systemic inflammation}

Inflammation has been epidemiologically and mechanistically linked to HFpEF (100). Broadly identified as a non-specific defensive response of the organism to threats, inflammatory pathways are active in HFpEF and, importantly, contribute to its pathogenesis.

$\mathrm{HFpEF}$ is a syndrome driven by multiple comorbidities and conditions (27). Virtually, all of these (e.g., obesity, hypertension, aging etc.) are characterized by a status of subtle, chronic systemic inflammation contributing to the overall complex inflammatory activation in HFpEF. Although both the clinical significance and specific pathways of inflammatory alterations in $\mathrm{HFpEF}$ are yet to be fully determined, accumulating clinical and experimental evidence suggest that immune dysregulation is critical in the pathophysiology of HFpEF.

\section{Clinical evidence of HFpEF as inflammatory disease}

Some of the most striking evidence supporting a role for inflammation as key element of HFpEF progression are based on circulating biomarker discovery. Classical biomarkers of myocyte stretch and necrosis (NP and troponins) have less diagnostic value in $\mathrm{HFpEF}$, compared to HFrEF (101). On the other hand, increased circulating levels of canonical inflammatory cytokines [e.g., tumor necrosis factor-alpha, soluble tumor necrosis factor-receptor I (sTNFRI), interleukin (IL)-6 and IL-1] were found to be predictive of incident HFpEF but not HFrEF (102-104). More recently, a European network analysis of circulating biomarkers in HF subjects revealed a unique biomarker preponderance related to inflammatory response, leukocyte 
migration and cell adhesion in HFpEF patients (105).

Multiple phenotypes of HFpEF can be identified and categorized in different phenogroups that only partially overlap in terms of prevalence of comorbidities, pathophysiological mechanisms and clinical outcomes. The use of machine learning techniques has recently allowed the identification of distinctive biomarker profiles in different HFpEF phenogroups $(102,106)$. Of interest, the presence of metabolic alterations seems to identify the phenogroup(s) with the greatest inflammatory activation. Specifically, increased adiposity has been associated with clusters of biomarkers related to immune activation and inflammation in specific HFpEF subgroups. The increase in circulating biomarkers of inflammation in $\mathrm{HFpEF}$ is supportive of the multiorgan, systemic inflammatory involvement in this syndrome. In addition, inflammatory activation (i.e., infiltrating immune cells and increased levels of critical mediators of inflammation) was demonstrated in $\mathrm{HFpEF}$ endomyocardial biopsies $(30,107)$.

Based on these findings, inhibition of inflammation has been explored as therapeutic strategy in HFpEF. For example, blockade of IL-1 signaling in HFpEF with anakinra has been tested in the D-HART and D-HART2 clinical studies. While in the pilot D-HART study, HFpEF patients treated with anakinra showed improved aerobic exercise capacity (108), these findings were not confirmed in the subsequent phase II study (109). Failure of this anticytokine therapeutic approach might be attributable to the heterogeneity of unselected HFpEF population. The accurate phenotypic sub-classification of $\mathrm{HFpEF}$ patients together with the characterization of specific inflammatory pathways active in different HFpEF subgroups will be critical to identify clusters of HFpEF patients that may benefit most from anti-inflammatory therapeutic approaches.

\section{Inflammatory-driven pathogenic pathways in HFpEF}

The clinical evidence for $\mathrm{HFpEF}$ as an inflammatory disease is corroborated by preclinical studies focusing on the mechanistic aspects of this syndrome. Notably, the current knowledge is limited to inflammatory mediators and aspects of innate immunity, whereas the involvement and extent of alterations of adaptive immunity in HFpEF is still largely unknown (110).

In HFpEF, inflammation has been implicated in promoting coronary microvascular endothelial dysfunction and in the perturbation of NO availability, two hallmarks of myocardial alterations in HFpEF. Increased expression of adhesion molecules in the inflamed coronary endothelium drives the recruitment and infiltration of macrophages and other inflammatory cells promoting the development of interstitial fibrosis, which together with cardiomyocyte stiffening, contributes to diastolic dysfunction in HFpEF (111). For example, hypertensioninduced diastolic dysfunction in mice causes the expansion of cardiac macrophage pools associated with the increased production of pro-fibrotic IL-10 which, in turn, amplifies and perpetuates diastolic dysfunction (112). Yet, the precise molecular events triggering the contribution of immune cells to diastolic dysfunction in HFpEF are still unknown.

Comorbidities-driven inflammation in $\mathrm{HFpEF}$ severely impacts on NO bioavailability in this syndrome. Inflammation increases oxidative stress and the production of ROS in HFpEF hearts, converting, in part, NO into peroxynitrite and other NO-related reactive species. In line with this notion, the presence of nitrosative stress in $\mathrm{HFpEF}$ has been identified as a major driver of this syndrome (113) (Figure 1).

The inflammatory-dependent reduction of $\mathrm{NO}$ bioavailability lowers the intracellular levels of cGMP, resulting in cardiomyocyte stiffening and impaired cellular relaxation (see "the role of titin in impaired cardiomyocyte relaxation" and "cGMP_signaling" sections).

Dysregulated NO production in HFpEF also involves the upregulation of inducible NOS (iNOS), a major inflammatory mediator, as shown in the myocardium of clinically relevant model(s) of $\mathrm{HFpEF}$ as well as in $\mathrm{HFpEF}$ human endomyocardial biopsies $(72,107)$. The resulting nitrosative stress impacts on cardiomyocyte function at many levels perturbing fundamental signaling pathways involved in cardiomyocyte responses to chronic stress (Figure 1). Interestingly, the role of iNOS in promoting cardiomyocyte dysfunction has been associated with metabolic syndrome and hypertension, comorbidities present in the majority of HFpEF patients. This suggests iNOS as a crucial effector of metabolic stress-induced inflammation-i.e., metabolic inflammation or metainflammation - and thus, as a common mechanism between HFpEF and other metabolic diseases that might present a valuable therapeutic target across many inflammatory-driven disorders.

\section{Neurobumoral activation}

A recent clinical study suggests that neurohumoral activation-as quantified by elevated plasma renin activity, aldosterone or catecholamine levels-is less prevalent 


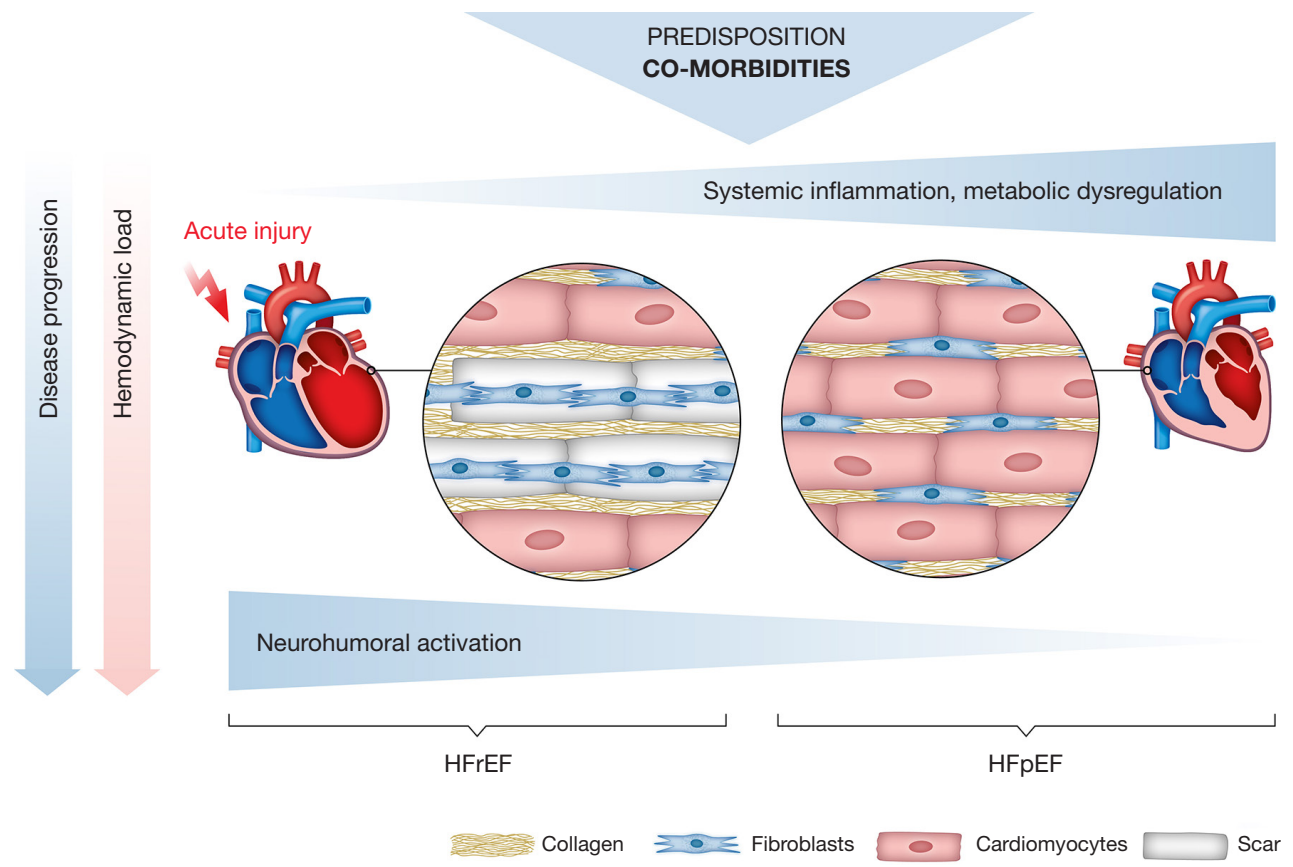

Figure 2 Myocardial remodeling in HFrEF and HFpEF. HFrEF, heart failure with reduced ejection fraction; HFpEF, heart failure with preserved ejection fraction.

in HFpEF vs. HFrEF (114). Similarly, HFpEF was not associated with significant RAS activation as measured by ACE activity (94). These observations agree with earlier results indicating that neurohumoral activation (RAS and sympathetic) in HF is related to the degree of reduction in LV EF $(115,116)$. Along similar lines, plasma aldosterone levels correlate with LV hypertrophy but not (independently) with LV diastolic dysfunction in patients with arterial hypertension (117). These findings may in part explain why RAS antagonists have failed to achieve survival benefit in HFpEF clinical trials $(4,118)$. Interestingly, despite effectively reducing arterial blood pressure, RAS-inhibition is also not associated with improved LV remodeling in patients with preserved EF (119). Taken together, current evidence suggests that RAS activation may be present in some HFpEF patients but is likely not a relevant driver for diastolic dysfunction or increased morbidity and mortality (Figure 2). The role of aldosterone antagonists in HFpEF, however, remains to be determined (120).

In line with above findings on neurohumoral activation, betablockers have not been found beneficial in clinical studies in HFpEF patients, and maybe even harmful by worsening impaired chronotropic responses in some patients (121). In fact, withdrawal of pre-existing betablocker therapy in HFpEF patients is currently investigated as a therapeutic approach (122).

\section{cGMP—signaling}

In HFpEF, increased myocardial passive stiffness, in large parts mediated by the sarcomeric protein titin, has been associated with reduced activity of the cGMP-protein kinase G-signaling cascade in cardiomyocytes. Physiologically NO (from intra- and extracellular sources, Figure 1) and NP stimulate guanylyl cyclases (GCs) to maintain cGMP homeostasis. Increased levels of NP are considered a protective response in HF (123). In HFpEF, however, reduced bioavailability of $\mathrm{NO}$, reduced efficacy of $\mathrm{NP}$, and direct impairment of GC function (by oxidative stress) synergize to cause an intracellular lack of cGMP. Based on encouraging experimental data, several pharmacological approaches to increase cGMP were evaluated in clinical trials: soluble GC stimulators/activators, NO donors, inhibitors of the degradation of either cGMP (PDE5-inhibitors) or BNP (sacubitril/valsartan). To date, none of these clinical approaches has resulted in prognostic benefits in $\mathrm{HFpEF}$ patients, but stimulation of the cGMP-PKG-dependent pathway remains an attractive strategy as discussed in (124). 


\section{Right ventricular dysfunction (RVD) in HFpEF}

\section{Clinical prevalence and prognostic value}

RVD, often in conjunction with pulmonary hypertension $(\mathrm{PH})$, is commonly observed in patients suffering from HFpEF and closely related to worse clinical outcomes evident as increased hospitalization rates and a considerable increase in overall mortality as compared to HFpEF patients without RVD (125-127) (for an in depth review on RVD mortality see also Sanders et al. in this issue, doi: 10.21037/cdt-20-450). Therefore, detailed and longitudinal $\mathrm{RV}$ phenotyping of HFpEF patients is warranted to identify individuals at higher risk for adverse cardiac events or on a declining trajectory.

In the past, only a few studies have systematically screened for RVD in patients with HFpEF with considerable variation in reported prevalence between and even within studies. Puwanant and colleagues detected impaired RV function, defined as a decline in echocardiographicallyassessed RV fractional area change (FAC) below $45 \%$, in approximately $33 \%$ of a total of $51 \mathrm{HFpEF}$ patients (128). Yet, when the authors defined RVD as tricuspid annular plane systolic excursion (TAPSE) of $<15 \mathrm{~mm}$ or peak systolic tricuspid annular tissue velocity S' $<115 \mathrm{~mm} / \mathrm{s}$, which both assess RV systolic performance, $40 \%$ and $50 \%$ of HFpEF patients, respectively, were classified with RVD (128). A larger study of $309 \mathrm{HFpEF}$ patients, however, reported RVD in only $20 \%$ of HFpEF patients, albeit using a slightly higher TAPSE of $<15.9 \mathrm{~mm}$ as cut-off (129). In yet another cohort of $201 \mathrm{HFpEF}$ patients, speckle-tracking echocardiography-derived RV strain identified RV systolic and diastolic abnormalities in $75 \%$ and $48 \%$ of patients, respectively (130). Finally, in a communitybased study from Olmsted County, Minnesota, of 500 patients identified with HFpEF 35\% and 21\% showed signs of RVD as defined by a TAPSE of $<16.0 \mathrm{~mm}$ or by semiquantitative two-dimensional echocardiography (i.e., visual assessment of RV contractility, and comparison of RV to LV size, summarized on a four-point ordinal scale) (131).

The considerable variability between studies in the reported prevalence can be in part attributed to differences in study populations (age, sex, comorbidities, medications), deviating study protocols for clinical assessment of RVD, and use of different cut-off values (132). While these variabilities preclude a precise estimate of the prevalence of RVD in HFpEF patients, one can conclude that RVD is a common comorbidity in HFpEF, with an average prevalence of $30 \%$ based on TAPSE criteria. As such, RVD should deserve special attention in HF phenotyping (133).

Importantly, in all of the above studies RVD was diagnosed based on echocardiographic assessment of RV morphology and function, rather than invasive measurements of systolic, mean, and diastolic pulmonary artery pressure (PAP) as well as pulmonary capillary wedge pressure (PCWP) as a direct reflection of increased RV afterload, to which the RV is particularly vulnerable. Although the etiology of increased RV afterload in HFpEF initially is primarily attributable to increased left-atrial (LA) filling pressures and subsequent retrograde congestion of the pulmonary veins, capillaries and arteries, the resulting $\mathrm{PH}$ is frequently complicated by secondary pulmonary vascular remodeling (134) (for discussion of the underlying mechanisms please see below). As such, assessment of the transpulmonary pressure gradient (TPG; mean PAPPCWP) or diastolic pressure gradient (DPG; diastolic PAPPCWP) constitutes an important diagnostic test for left and right heart disease (135). Accordingly, state-of-the-art diagnostic HFpEF guidelines recommend the assessment of PCWP for a subgroup of patients as part of a diastolic stress testing (136). Given the risks associated with right heart catheterization, invasive hemodynamic phenotyping of the right heart is, however, scarcely performed. Indeed, simultaneous left and right heart hemodynamics may be warranted in some patients as outlined by Hansmann et al. in this issue (doi: 10.21037/cdt-20-483).

The prognostic relevance of RVD in HFpEF patients was highlighted by the group of Margaret Redfield in the already mentioned Olmsted County study (131) demonstrating that all-cause and cardiovascular mortality, as well as time to first or multiple HF hospitalizations are associated with increased systolic PAP, decreased TAPSE, and semi-quantitative confirmation of RVD. These findings were corroborated in a subsequent study which reported an almost 10-fold higher 1-year all-cause mortality for HFpEF patients with RVD compared to those without (41.7\% vs. $4.8 \%$ ) (127). On the basis of these data, it seems compelling that HF phenotyping guidelines need to implement standardized RV phenotyping protocols to identify patient populations who evidently face the greatest risk for critical events.

\section{Potential mechanisms of $R V$ dysfunction}

HFpEF causes "passive" backward congestion of blood pressure from the left ventricle into the pulmonary circulation and ultimately, the RV; however, with 

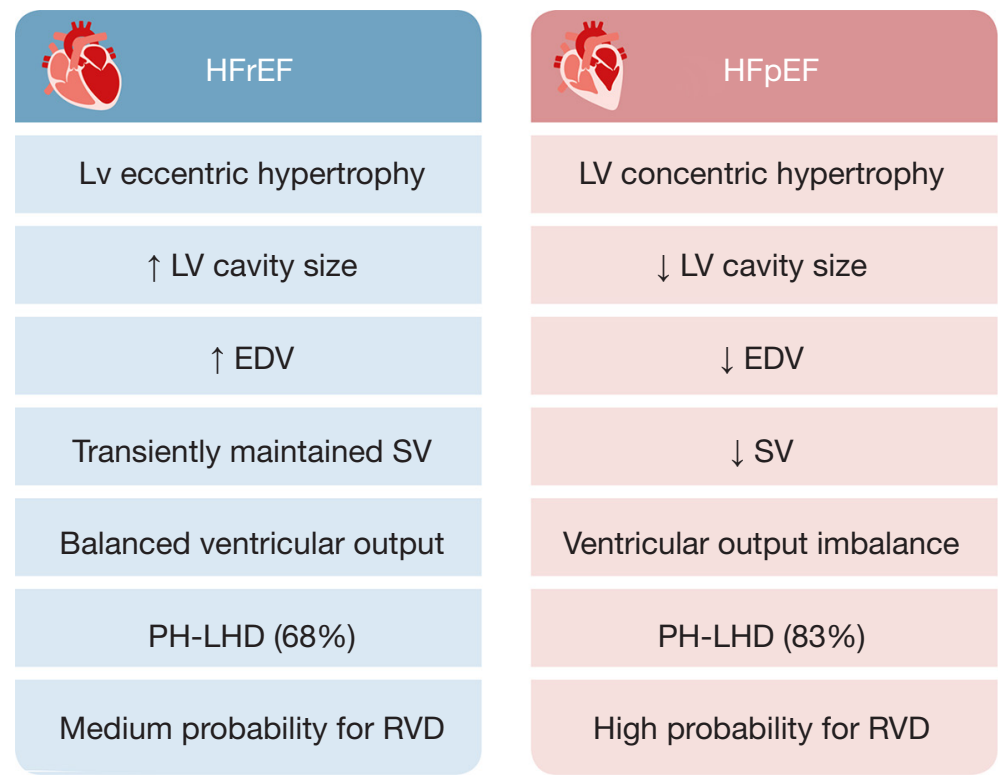

Figure 3 RVD in HFpEF vs. HFrEF. HFpEF is characterized by concentric LV hypertrophy. In turn, LV cavity size, EDV, and ultimately SV decrease. The resulting ventricular output imbalance between LV and RV increases the prevalence of PH-LHD and hence, the probability of RVD. Conversely, HFrEF causes LV dilatation, increasing LV cavity size and EDV. This enables the LV to transiently maintain a stable SV despite a reduced EF. As a consequence, ventricular output imbalance, PH-LHD and ultimately RVD are less common in HFrEF. RVD, right ventricular dysfunction; HFpEF, heart failure with preserved ejection fraction; HFrEF, heart failure with reduced ejection fraction; LV, left ventricular; EDV, end-diastolic volume; SV, stroke volume; PH-LHD, pulmonary hypertension associated with left heart disease.

advancing HFpEF severity and duration additional "active" mechanisms such as lung vascular remodeling and LV-RV interaction come progressively into play. While normal $\mathrm{RV}$ function is often maintained during mere passive congestion-frequently referred to as "isolated postcapillary $\mathrm{PH}$ " (IpcPH) —additional "active" pulmonary vascular remodeling will result in "combined post- and pre-capillary $\mathrm{PH}$ " $(\mathrm{CpcPH})$ which directly determines the extent and course of RVD $(137,138)$.

\section{Postcapillary pressure overload}

The "passive" component of backwards congestion in left heart disease (LHD) is based on the basic physical principle of pressure transmission along communicating pipes that ultimately results in $\mathrm{PH}$. While $\mathrm{PH}$ was originally, yet somewhat arbitrarily, defined as mean PAP $\geq 25 \mathrm{mmHg}$, a series of seminal studies published in the past 5 years revealed that individuals with a mean $\mathrm{PAP}$ of 21 to $24 \mathrm{mmHg}$ are also at increased risk for poor outcomes $(139,140)$. At the 6th World Symposium for Pulmonary Hypertension in Nice in 2018, these findings led to the recommendation to define a mean $\mathrm{PAP} \geq 20 \mathrm{mmHg}$ as new cutoff for diagnosing
$\mathrm{PH}$ (141). Of the 5 different groups of $\mathrm{PH}$, the most common one is PH-LHD which in addition to an increase in mean PAP $\geq 20 \mathrm{mmHg}$ is defined by a concomitant PCWP $>15$ mmHg (142-144). Remarkably, epidemiological data suggest that PH-LHD (and thus, presumably, also RVD) is more frequent in HFpEF (83\%) (145) as compared to HFrEF patients (68\%) (146). This differential prevalence is in line with basic physiological principles (Figure 3): The hallmarks of HFpEF are concentric LV hypertrophy and diastolic dysfunction; in contrast, HFrEF is characterized by systolic dysfunction and $\mathrm{LV}$ dilatation. As a result, end-diastolic volumes (EDVs) are considerably smaller in HFpEF as compared to HFrEF, and even though LV EF will by definition be higher, the resulting LV SV will be smaller in HFpEF as compared to HFrEF (147). Importantly, it is the difference in SV, not in $\mathrm{EF}$ that characterizes ventricular output imbalance. Given the more pronounced reduction in LV SV, it may thus come as little surprise that PH-LHD is apparently more frequent in $\mathrm{HFpEF}$ as compared to HFrEF.

The current nomenclature classifies "passive" postcapillary pressure overload as $\mathrm{IpcPH}$, defined as $\mathrm{PH}$ - 
Table 2 Hemodynamic definitions of $\mathrm{PH}$

\begin{tabular}{cl}
\hline PH type & Hemodynamic definitions \\
\hline PH-LHD & mPAP $\geq 20 \mathrm{mmHg} ;$ PCWP $>15 \mathrm{mmHg}$ \\
IpcPH & PVR $\leq 3 \mathrm{WU} ; \mathrm{DPG}<7 \mathrm{mmHg} ; \mathrm{C}_{\mathrm{PA}} \geq 2.3 \mathrm{~mL} / \mathrm{mmHg}$ \\
$\mathrm{CpcPH}$ & $\mathrm{PVR}>3 \mathrm{WU} ; \mathrm{DPG} \geq 7 \mathrm{mmHg} ; \mathrm{C}_{\mathrm{PA}}<2.3 \mathrm{~mL} / \mathrm{mmHg}$ \\
\hline
\end{tabular}

Modified from (138). PH-LHD, pulmonary hypertension associated with left heart disease; mPAP, mean pulmonary artery pressure; PCWP, pulmonary capillary wedge pressure; $\mathrm{IpcPH}$, isolated post-capillary pulmonary hypertension; $\mathrm{CpcPH}$, combined post- and precapillary pulmonary hypertension; PVR, pulmonary vascular resistance; WU, Wood units; DPG, diastolic pressure gradient; CPA, pulmonary artery compliance.

LHD with a pulmonary vascular resistance (PVR) $\leq 3$ Wood units, a DPG $<7 \mathrm{mmHg}$, or a pulmonary artery compliance $\geq 2.3 \mathrm{~mL} / \mathrm{mmHg}$ (Table 2). Pathologically, IpcPH is primarily characterized by the typical signs of congestive HF, i.e., interstitial and subsequent alveolar lung oedema, which in case of capillary stress failure can advance to alveolar hemorrhage (148).

\section{Precapillary PH}

When "active" pulmonary vascular remodelling adds a precapillary component to the post-capillary pressure overload, IpcPH transitions to $\mathrm{CpcPH}$ defined as $\mathrm{PH}$ LHD with a PVR $>3$ Wood units, a DPG $\geq 7 \mathrm{mmHg}$, or a pulmonary artery compliance $<2.3 \mathrm{~mL} / \mathrm{mmHg}$ (Table 1). The underlying changes in the pulmonary vasculature comprise both medial and intimal thickening across the entirety of small vessels in the lung $(134,149,150)$. The combined "passive" and "active" effects of HFpEF and pulmonary vascular remodeling ultimately affect the function of the $\mathrm{RV}$ (for a comprehensive overview on the non-invasive and invasive assessment of RV hemodynamics see Bernardo et al. and Truog et al. in this issue, doi: 10.21037/cdt-20479, doi: 10.21037/cdt-20-272). In response to the elevated afterload, the RV undergoes compensatory concentric hypertrophy and at its peak, is able to increase contractility by a factor of five (144). This phenomenon is commonly described as RV-PA coupling, which describes the energy transfer between RV contractility and arterial afterload, where RV contractility can be characterized by end-systolic elastance (Ees). Arterial afterload can be thought of as net arterial stiffness (Ea) $(151,152)$. With disease progression, intraventricular pressure rises exerting increased stress on the RV wall inevitably leading to RV dilation. Increasing oxygen demand (required to pump against an increased afterload) can no longer be matched by a decreasing oxygen supply as diastolic coronary perfusion is progressively reduced as a result of increased ventricular wall tension. In combination, these events trigger a downward spiral of ischemia, deteriorating contractility, failing RV CO and ultimately general decompensation (142).

\section{$L V-R V$ interaction}

An additional characteristic feature of RVD in HFpEF patients is tricuspid valve (TV) regurgitation due to enlargement of the TV annulus - preferably at the level of the free wall as a consequence of RV dilation (153). Similarly or in parallel, regurgitation may be caused by papillary muscle displacement as a result of RV remodeling (154). Finally, TV regurgitation may be directly driven by pathological changes in cardiac geometry: The transmission of LV structural and locomotorical changes via the interventricular septum to the RV increases the risk of TV leaflet prolapse and affects the position of the septal leaflet up to a point where efficient valve coaptation is no longer possible $(155,156)$. The resulting TV regurgitation will further contribute to RV dilation and impaired RV $\mathrm{CO}$, thus adding to the vicious cycle of accelerating $\mathrm{RV}$ failure. The latter scenario also underlines that in addition to "passive" congestion and "active" pulmonary vascular remodeling, direct interaction between the RV and $\mathrm{LV}$ may drive RVD in HFpEF. Conversely, the progressive increase in $\mathrm{RV}$ afterload in $\mathrm{CpcPH}$ may further negatively impact on LV diastolic function, as indicated by the clinical finding that release of RV afterload by pulmonary thrombarterectomy markedly improves LV EDV and SV (157). This RV-LV interaction is probably mediated in large part through septal interdependence, as leftward displacement of the interventricular septum further impedes LV filling (158). Notably, RV-LV interdependence extends beyond the level of hemodynamic interactions (158), but may comprise local remodeling and pro-fibrotic effects at the ventricular hinge-points (159) as well as vascular information transfer via gap junction-mediated conducted responses that can propagate to the contralateral ventricle along the continuity of the shared RV-LV microvasculature.

\section{Future molecular approaches to HFpEF}

As outlined in this review, current clinical and experimental evidence supports the concept of inflammation contributing to HFpEF pathogenesis. However, a better understanding 
of targetable signaling pathways is needed to translate these discoveries into novel therapeutic approaches. To this end, the careful phenotyping of clinically relevant animal models in vivo and in vitro, both at rest and during exercise/stress is mandatory in order to characterize a functional response rather than a steady state. While chronic inflammation sets the stage for cardiac remodeling, different co-morbidities likely trigger specific patterns of cellular adaptations which need to be delineated preferably in a structured comparison of animal models. Targeting well established pathways (e.g., cGMP-PKG) calls for novel approaches [such as inhibition of different PDEs (160) or sGLT inhibitors (161)]. Efforts to exploit identified mitochondrial-based therapeutic approaches (anti-ROS, pro ATP) are already underway (85). Cardiomyocyte ECC is tightly controlled and interventions targeting intracellular $\mathrm{Na}^{+}$and $\mathrm{Ca}^{2+}$ need to take chronic adaptation of ion homeostasis into account (52). Myocardial fibrosis-while prevalent in $\mathrm{HFpEF}$ - requires validation as therapeutic target using established (spironolactone) and novel substances with anti-fibrotic activity (162). Promoting a targeted therapeutic approach (6), a more in-depth clinical phenotyping including comprehensive imaging analysis of both ventricles (strain, LA and RV involvement) and multibiomarker panels $(163,164)$ will be the basis for HFpEF treatment with the goal to identify phenogroups of patients who may have the highest likelihood to benefit from a specific targeted therapy.

\section{Acknowledgments}

Funding: Dr. FH is supported by the Else-KrönerFresenius Stiftung and by the BMBF (German Ministry of Education and Research). Drs. FH and UP are funded by the BIH-Charité Clinical Scientist Program of CharitéUniversitätsmedizin Berlin and the Berlin Institute of Health. The UMCG, which employs Dr. RA de Boer has received research grants and/or fees from AstraZeneca, Abbott, Bristol-Myers Squibb, Novartis, Novo Nordisk, and Roche. Dr. RA de Boer received speaker fees from Abbott, AstraZeneca, Novartis, and Roche. Dr. WMK is funded by Heart \& Stroke Foundation of Canada, DZHK (German Center for Cardiovascular Research), DFG (German Research Foundation), and BMBF (German Ministry for Education and Research).

\section{Footnote}

Provenance and Peer Review: This article was commissioned by the Guest Editors (Martin Koestenberger, HarmJan Bogaard and Georg Hansmann) for the series "Right Ventricular Dysfunction" published in Cardiovascular Diagnosis and Therapy. The article was sent for external peer review organized by the Editor-in-Chief and the editorial office.

Conflicts of Interest: All authors have completed the ICMJE uniform disclosure form (available at http://dx.doi. org/10.21037/cdt-20-477). The series "Right Ventricular Dysfunction" was commissioned by the editorial office without any funding or sponsorship. Dr. RA de Boer reports grants from Abbott, grants from AstraZeneca, grants from Bristol-Myers Squibb, grants from Novartis, grants from NovoNordisk, grants from Roche, personal fees from Abbott, personal fees from AstraZeneca, personal fees from NovoNordisk, personal fees from Roche, outside the submitted work. Dr. WMK reports grants from Heart and Stroke Foundation of Canada, grants from German Center for Cardiovascular Research, grants from German Research Foundation, grants from German Ministry for Education and Research, during the conduct of the study. The authors have no other conflicts of interest to declare.

Ethical Statement: The authors are accountable for all aspects of the work in ensuring that questions related to the accuracy or integrity of any part of the work are appropriately investigated and resolved.

Open Access Statement: This is an Open Access article distributed in accordance with the Creative Commons Attribution-NonCommercial-NoDerivs 4.0 International License (CC BY-NC-ND 4.0), which permits the noncommercial replication and distribution of the article with the strict proviso that no changes or edits are made and the original work is properly cited (including links to both the formal publication through the relevant DOI and the license). See: https://creativecommons.org/licenses/by-nc-nd/4.0/.

\section{References}

1. Lam CSP, Gamble GD, Ling LH, et al. Mortality associated with heart failure with preserved vs. reduced ejection fraction in a prospective international multi-ethnic cohort study. Eur Heart J 2018;39:1770-80.

2. Ambrosy AP, Fonarow GC, Butler J, et al. The global health and economic burden of hospitalizations for heart failure: lessons learned from hospitalized heart failure 
registries. J Am Coll Cardiol 2014;63:1123-33.

3. Ponikowski P, Voors AA, Anker SD, et al. 2016 ESC Guidelines for the diagnosis and treatment of acute and chronic heart failure: The Task Force for the diagnosis and treatment of acute and chronic heart failure of the European Society of Cardiology (ESC) Developed with the special contribution of the Heart Failure Association (HFA) of the ESC. Eur Heart J 2016;37:2129-200.

4. Martin N, Manoharan K, Thomas J, et al. Beta-blockers and inhibitors of the renin-angiotensin aldosterone system for chronic heart failure with preserved ejection fraction. Cochrane Database Syst Rev 2018;6:CD012721.

5. Kjeldsen SE, von Lueder TG, Smiseth OA, et al. Medical therapies for heart failure with preserved ejection fraction. Hypertension 2020;75:23-32.

6. Senni M, Paulus WJ, Gavazzi A, et al. New strategies for heart failure with preserved ejection fraction: the importance of targeted therapies for heart failure phenotypes. Eur Heart J 2014;35:2797-815.

7. Kao DP, Lewsey JD, Anand IS, et al. Characterization of subgroups of heart failure patients with preserved ejection fraction with possible implications for prognosis and treatment response. Eur J Heart Fail 2015;17:925-35.

8. Khan MS, Fonarow GC, Khan H, et al. Renin-angiotensin blockade in heart failure with preserved ejection fraction: a systematic review and meta-analysis. ESC Heart Fail 2017;4:402-8.

9. Streng KW, Nauta JF, Hillege HL, et al. Non-cardiac comorbidities in heart failure with reduced, mid-range and preserved ejection fraction. Int J Cardiol 2018;271:132-9.

10. Iorio A, Senni M, Barbati G, et al. Prevalence and prognostic impact of non-cardiac co-morbidities in heart failure outpatients with preserved and reduced ejection fraction: a community-based study. Eur J Heart Fail 2018;20:1257-66.

11. Mentz RJ, Kelly JP, von Lueder TG, et al. Noncardiac comorbidities in heart failure with reduced versus preserved ejection fraction. J Am Coll Cardiol 2014;64:2281-93.

12. Del Re DP, Amgalan D, Linkermann A, et al. Fundamental mechanisms of regulated cell death and implications for heart disease. Physiol Rev 2019;99:1765-817.

13. de Boer RA, De Keulenaer G, Bauersachs J, et al. Towards better definition, quantification and treatment of fibrosis in heart failure. A scientific roadmap by the Committee of Translational Research of the Heart Failure Association (HFA) of the European Society of Cardiology. Eur J Heart Fail 2019;21:272-85.
14. Packer M. The neurohormonal hypothesis: a theory to explain the mechanism of disease progression in heart failure. J Am Coll Cardiol 1992;20:248-54.

15. Braunwald E, Pfeffer MA. Ventricular enlargement and remodeling following acute myocardial infarction: mechanisms and management. Am J Cardiol 1991;68:1D-6D.

16. Simmonds SJ, Cuijpers I, Heymans S, et al. Cellular and molecular differences between HFpEF and HFrEF: a step ahead in an improved pathological understanding. Cells 2020;9:242.

17. Ton VK, Mukherjee M, Judge DP. Transthyretin cardiac amyloidosis: pathogenesis, treatments, and emerging role in heart failure with preserved ejection fraction. Clin Med Insights Cardiol 2015;8:39-44.

18. Anselmi A, Gaudino M, Baldi A, et al. Role of apoptosis in pressure-overload cardiomyopathy. J Cardiovasc Med (Hagerstown) 2008;9:227-32.

19. van Heerebeek L, Borbely A, Niessen HW, et al. Myocardial structure and function differ in systolic and diastolic heart failure. Circulation 2006;113:1966-73.

20. Maron BJ, Rowin EJ, Udelson JE, et al. Clinical spectrum and management of heart failure in hypertrophic cardiomyopathy. JACC Heart Fail 2018;6:353-63.

21. Marian AJ, Braunwald E. Hypertrophic cardiomyopathy: genetics, pathogenesis, clinical manifestations, diagnosis, and therapy. Circ Res 2017;121:749-70.

22. Sen-Chowdhry S, Jacoby D, Moon JC, et al. Update on hypertrophic cardiomyopathy and a guide to the guidelines. Nat Rev Cardiol 2016;13:651-75.

23. Franssen C, Gonzalez Miqueo A. The role of titin and extracellular matrix remodelling in heart failure with preserved ejection fraction. Neth Heart J 2016;24:259-67.

24. Zile MR, Baicu CF, Ikonomidis JS, et al. Myocardial stiffness in patients with heart failure and a preserved ejection fraction: contributions of collagen and titin. Circulation 2015;131:1247-59.

25. Campbell KS, Sorrell VL. Cell- and molecular-level mechanisms contributing to diastolic dysfunction in HFpEF. J Appl Physiol (1985) 2015;119:1228-32 .

26. Su MY, Lin LY, Tseng YH, et al. CMR-verified diffuse myocardial fibrosis is associated with diastolic dysfunction in HFpEF. JACC Cardiovasc Imaging 2014;7:991-7.

27. Shah SJ, Kitzman DW, Borlaug BA, et al. Phenotypespecific treatment of heart failure with preserved ejection fraction: a multiorgan roadmap. Circulation 2016;134:73-90. 
28. Mohammed SF, Hussain S, Mirzoyev SA, et al. Coronary microvascular rarefaction and myocardial fibrosis in heart failure with preserved ejection fraction. Circulation 2015;131:550-9.

29. Hamdani N, Franssen C, Lourenco A, et al. Myocardial titin hypophosphorylation importantly contributes to heart failure with preserved ejection fraction in a rat metabolic risk model. Circ Heart Fail 2013;6:1239-49.

30. Kasner M, Westermann D, Lopez B, et al. Diastolic tissue Doppler indexes correlate with the degree of collagen expression and cross-linking in heart failure and normal ejection fraction. J Am Coll Cardiol 2011;57:977-85.

31. van Heerebeek L, Hamdani N, Handoko ML, et al. Diastolic stiffness of the failing diabetic heart: importance of fibrosis, advanced glycation end products, and myocyte resting tension. Circulation 2008;117:43-51.

32. Anderson MM, Requena JR, Crowley JR, et al. The myeloperoxidase system of human phagocytes generates Nepsilon-(carboxymethyl)lysine on proteins: a mechanism for producing advanced glycation end products at sites of inflammation. J Clin Invest 1999;104:103-13.

33. van Deel ED, Najafi A, Fontoura D, et al. In vitro model to study the effects of matrix stiffening on $\mathrm{Ca}(2+)$ handling and myofilament function in isolated adult rat cardiomyocytes. J Physiol 2017;595:4597-610.

34. Bang ML, Centner T, Fornoff F, et al. The complete gene sequence of titin, expression of an unusual approximately $700-\mathrm{kDa}$ titin isoform, and its interaction with obscurin identify a novel Z-line to I-band linking system. Circ Res 2001;89:1065-72.

35. Krüger M, Kötter S, Grützner A, et al. Protein kinase G modulates human myocardial passive stiffness by phosphorylation of the titin springs. Circ Res 2009;104:87-94.

36. Franssen C, Chen S, Unger A, et al. Myocardial microvascular inflammatory endothelial activation in heart failure with preserved ejection fraction. JACC Heart Fail 2016;4:312-24.

37. van Heerebeek L, Franssen CP, Hamdani N, et al. Molecular and cellular basis for diastolic dysfunction. Curr Heart Fail Rep 2012;9:293-302.

38. Borlaug BA, Paulus WJ. Heart failure with preserved ejection fraction: pathophysiology, diagnosis, and treatment. Eur Heart J 2011;32:670-9.

39. Lourenço AP, Leite-Moreira AF, Balligand JL, et al. An integrative translational approach to study heart failure with preserved ejection fraction: a position paper from the Working Group on Myocardial Function of the European Society of Cardiology. Eur J Heart Fail 2018;20:216-27.

40. Curl CL, Danes VR, Bell JR, et al. Cardiomyocyte functional etiology in heart failure with preserved ejection fraction is distinctive-a new preclinical model. J Am Heart Assoc 2018;7:e007451.

41. Adeniran I, MacIver DH, Hancox JC, et al. Abnormal calcium homeostasis in heart failure with preserved ejection fraction is related to both reduced contractile function and incomplete relaxation: an electromechanically detailed biophysical modeling study. Front Physiol 2015;6:78.

42. Sag CM, Wagner S, Maier LS. Role of oxidants on calcium and sodium movement in healthy and diseased cardiac myocytes. Free Radic Biol Med 2013;63:338-49.

43. Kranstuber AL, Del Rio C, Biesiadecki BJ, et al. Advanced glycation end product cross-link breaker attenuates diabetes-induced cardiac dysfunction by improving sarcoplasmic reticulum calcium handling. Front Physiol 2012;3:292.

44. Schober T, Huke S, Venkataraman R, et al. Myofilament Ca sensitization increases cytosolic Ca binding affinity, alters intracellular Ca homeostasis, and causes pause-dependent Ca-triggered arrhythmia. Circ Res 2012;111:170-9.

45. Bers DM. Excitation-contraction coupling and cardiac contractile force. 2nd ed. Amsterdam: Kluwer Academic Publishers, 2001.

46. Hasenfuss G, Schillinger W, Lehnart SE, et al. Relationship between $\mathrm{Na}+-\mathrm{Ca} 2+-$ exchanger protein levels and diastolic function of failing human myocardium. Circulation 1999;99:641-8.

47. Primessnig U, Schonleitner P, Holl A, et al. Novel pathomechanisms of cardiomyocyte dysfunction in a model of heart failure with preserved ejection fraction. Eur J Heart Fail 2016;18:987-97.

48. Pieske B, Houser SR. [Na+]i handling in the failing human heart. Cardiovasc Res 2003;57:874-86.

49. Despa S, Bers DM. Na(+) transport in the normal and failing heart - remember the balance. J Mol Cell Cardiol 2013;61:2-10.

50. Eisner DA, Caldwell JL, Trafford AW, et al. The control of diastolic calcium in the heart: basic mechanisms and functional implications. Circ Res 2020;126:395-412.

51. Heinzel FR, Hohendanner F, Jin G, et al. Myocardial hypertrophy and its role in heart failure with preserved ejection fraction. J Appl Physiol (1985) 2015;119:1233-42.

52. Primessnig U, Bracic T, Levijoki J, et al. Long-term 
effects of $\mathrm{Na}(+) / \mathrm{Ca}(2+)$ exchanger inhibition with ORM-11035 improves cardiac function and remodelling without lowering blood pressure in a model of heart failure with preserved ejection fraction. Eur J Heart Fail 2019;21:1543-52.

53. Rouhana S, Farah C, Roy J, et al. Early calcium handling imbalance in pressure overload-induced heart failure with nearly normal left ventricular ejection fraction. Biochim Biophys Acta Mol Basis Dis 2019;1865:230-42.

54. Sossalla S, Wagner S, Rasenack EC, et al. Ranolazine improves diastolic dysfunction in isolated myocardium from failing human hearts--role of late sodium current and intracellular ion accumulation. J Mol Cell Cardiol 2008;45:32-43.

55. Undrovinas NA, Maltsev VA, Belardinelli L, et al. Late sodium current contributes to diastolic cell $\mathrm{Ca} 2+$ accumulation in chronic heart failure. J Physiol Sci 2010;60:245-57.

56. Coppini R, Ferrantini C, Yao L, et al. Late sodium current inhibition reverses electromechanical dysfunction in human hypertrophic cardiomyopathy. Circulation 2013;127:575-84.

57. De Angelis A, Cappetta D, Piegari E, et al. Longterm administration of ranolazine attenuates diastolic dysfunction and adverse myocardial remodeling in a model of heart failure with preserved ejection fraction. Int J Cardiol 2016;217:69-79.

58. Maier LS, Layug B, Karwatowska-Prokopczuk E, et al. RAnoLazIne for the treatment of diastolic heart failure in patients with preserved ejection fraction: the RALI-DHF proof-of-concept study. JACC Heart Fail 2013;1:115-22.

59. Runte KE, Bell SP, Selby DE, et al. Relaxation and the role of calcium in isolated contracting myocardium from patients with hypertensive heart disease and heart failure with preserved ejection fraction. Circ Heart Fail 2017;10:e004311.

60. Verdonck F, Mubagwa K, Sipido KR. $[\mathrm{Na}(+)]$ in the subsarcolemmal 'fuzzy' space and modulation of $[\mathrm{Ca}(2+)]$ (i) and contraction in cardiac myocytes. Cell Calcium 2004;35:603-12.

61. Sachse FB, Clark R, Giles WR. No fuzzy space for intracellular $\mathrm{Na}(+)$ in healthy ventricular myocytes. J Gen Physiol 2017;149:683-7.

62. Ho CY, Mealiffe ME, Bach RG, et al. Evaluation of mavacamten in symptomatic patients with nonobstructive hypertrophic cardiomyopathy. J Am Coll Cardiol 2020;75:2649-60.
63. Ho CY, Olivotto I, Jacoby D, et al. Study design and rationale of EXPLORER-HCM: evaluation of mavacamten in adults with symptomatic obstructive hypertrophic cardiomyopathy. Circ Heart Fail 2020;13:e006853.

64. Heitner SB, Jacoby D, Lester SJ, et al. Mavacamten treatment for obstructive hypertrophic cardiomyopathy: a clinical trial. Ann Intern Med 2019;170:741-8.

65. Chung JH, Biesiadecki BJ, Ziolo MT, et al. Myofilament calcium sensitivity: role in regulation of in vivo cardiac contraction and relaxation. Front Physiol 2016;7:562.

66. Marston S, Zamora JE. Troponin structure and function: a view of recent progress. J Muscle Res Cell Motil 2020;41:71-89.

67. Gresham KS, Stelzer JE. The contributions of cardiac myosin binding protein $\mathrm{C}$ and troponin I phosphorylation to beta-adrenergic enhancement of in vivo cardiac function. J Physiol 2016;594:669-86.

68. Malik FI, Hartman JJ, Elias KA, et al. Cardiac myosin activation: a potential therapeutic approach for systolic heart failure. Science 2011;331:1439-43.

69. Green EM, Wakimoto H, Anderson RL, et al. A smallmolecule inhibitor of sarcomere contractility suppresses hypertrophic cardiomyopathy in mice. Science 2016;351:617-21.

70. Hamdani N, Bishu KG, von Frieling-Salewsky M, et al. Deranged myofilament phosphorylation and function in experimental heart failure with preserved ejection fraction. Cardiovasc Res 2013;97:464-71.

71. Tong CW, Nair NA, Doersch KM, et al. Cardiac myosinbinding protein-C is a critical mediator of diastolic function. Pflugers Arch 2014;466:451-7.

72. Rosas PC, Warren CM, Creed HA, et al. Cardiac myosin binding protein-C phosphorylation mitigates age-related cardiac dysfunction: hope for better aging? JACC Basic Transl Sci 2019;4:817-30.

73. Lovelock JD, Monasky MM, Jeong EM, et al. Ranolazine improves cardiac diastolic dysfunction through modulation of myofilament calcium sensitivity. Circ Res 2012;110:841-50.

74. Jeong EM, Monasky MM, Gu L, et al. Tetrahydrobiopterin improves diastolic dysfunction by reversing changes in myofilament properties. J Mol Cell Cardiol 2013;56:44-54.

75. Papadaki M, Holewinski RJ, Previs SB, et al. Diabetes with heart failure increases methylglyoxal modifications in the sarcomere, which inhibit function. JCI Insight 2018;3:e121264.

76. Haizlip KM, Hiranandani N, Biesiadecki BJ, et al. Impact 
of hydroxyl radical-induced injury on calcium handling and myofilament sensitivity in isolated myocardium. J Appl Physiol (1985) 2012;113:766-74.

77. Tsujimoto T, Kajio H. Abdominal obesity is associated with an increased risk of all-cause mortality in patients with HFpEF. J Am Coll Cardiol 2017;70:2739-49.

78. Rozenbaum Z, Topilsky Y, Khoury S, et al. Association of body mass index and diastolic function in metabolically healthy obese with preserved ejection fraction. Int J Cardiol 2019;277:147-52.

79. Oh A, Okazaki R, Sam F, et al. Heart failure with preserved ejection fraction and adipose tissue: a story of two tales. Front Cardiovasc Med 2019;6:110.

80. Maack C, Lehrke M, Backs J, et al. Heart failure and diabetes: metabolic alterations and therapeutic interventions: a state-of-the-art review from the Translational Research Committee of the Heart Failure Association-European Society of Cardiology. Eur Heart J 2018;39:4243-54.

81. Koutroumpakis E, Jozwik B, Aguilar D, et al. Strategies of unloading the failing heart from metabolic stress. Am J Med 2020;133:290-6.

82. Gupta A, Houston B. A comprehensive review of the bioenergetics of fatty acid and glucose metabolism in the healthy and failing heart in nondiabetic condition. Heart Fail Rev 2017;22:825-42.

83. De Jong KA, Lopaschuk GD. Complex energy metabolic changes in heart failure with preserved ejection fraction and heart failure with reduced ejection fraction. Can J Cardiol 2017;33:860-71.

84. Katz SD. Pathophysiology of chronic systolic heart failure. A view from the periphery. Ann Am Thorac Soc 2018;15:S38-41.

85. Kumar AA, Kelly DP, Chirinos JA. Mitochondrial dysfunction in heart failure with preserved ejection fraction. Circulation 2019;139:1435-50.

86. Fillmore N, Levasseur JL, Fukushima A, et al. Uncoupling of glycolysis from glucose oxidation accompanies the development of heart failure with preserved ejection fraction. Mol Med 2018;24:3.

87. Kessler G, Friedman J. Metabolism of fatty acids and glucose. Circulation 1998;98:1351.

88. Brandt MM, Nguyen ITN, Krebber MM, et al. Limited synergy of obesity and hypertension, prevalent risk factors in onset and progression of heart failure with preserved ejection fraction. J Cell Mol Med 2019;23:6666-78.

89. Charles CJ, Lee P, Li RR, et al. A porcine model of heart failure with preserved ejection fraction: magnetic resonance imaging and metabolic energetics. ESC Heart Fail 2020;7:92-102.

90. Zhang L, Jaswal JS, Ussher JR, et al. Cardiac insulinresistance and decreased mitochondrial energy production precede the development of systolic heart failure after pressure-overload hypertrophy. Circ Heart Fail 2013;6:1039-48.

91. Chaanine AH, Joyce LD, Stulak JM, et al. Mitochondrial morphology, dynamics, and function in human pressure overload or ischemic heart disease with preserved or reduced ejection fraction. Circ Heart Fail 2019;12:e005131.

92. Phan TT, Abozguia K, Nallur Shivu G, et al. Heart failure with preserved ejection fraction is characterized by dynamic impairment of active relaxation and contraction of the left ventricle on exercise and associated with myocardial energy deficiency. J Am Coll Cardiol 2009;54:402-9.

93. Silberman GA, Fan TH, Liu H, et al. Uncoupled cardiac nitric oxide synthase mediates diastolic dysfunction. Circulation 2010;121:519-28.

94. Negi SI, Jeong EM, Shukrullah I, et al. ReninAngiotensin activation and oxidative stress in early heart failure with preserved ejection fraction. Biomed Res Int 2015;2015:825027.

95. Clementi E, Nisoli E. Nitric oxide and mitochondrial biogenesis: a key to long-term regulation of cellular metabolism. Comp Biochem Physiol A Mol Integr Physiol 2005;142:102-10.

96. Bertero E, Maack C. Calcium signaling and reactive oxygen species in mitochondria. Circ Res 2018;122:1460-78.

97. Dorn GW, 2nd, Maack C. SR and mitochondria: calcium cross-talk between kissing cousins. J Mol Cell Cardiol 2013;55:42-9.

98. Chaanine AH, Sreekumaran Nair K, Bergen RH 3rd, et al. Mitochondrial integrity and function in the progression of early pressure overload-induced left ventricular remodeling. J Am Heart Assoc 2017;6:e05869.

99. Hiemstra JA, Veteto AB, Lambert MD, et al. Chronic lowintensity exercise attenuates cardiomyocyte contractile dysfunction and impaired adrenergic responsiveness in aortic-banded mini-swine. J Appl Physiol (1985) 2018;124:1034-44.

100. Paulus WJ, Tschope C. A novel paradigm for heart failure with preserved ejection fraction: comorbidities drive myocardial dysfunction and remodeling through coronary microvascular endothelial inflammation. J Am Coll Cardiol 
2013;62:263-71.

101.de Boer RA, Nayor M, deFilippi CR, et al. Association of cardiovascular biomarkers with incident heart failure with preserved and reduced ejection fraction. JAMA Cardiol 2018;3:215-24.

102. Chirinos JA, Orlenko A, Zhao L, et al. Multiple plasma biomarkers for risk stratification in patients with heart failure and preserved ejection fraction. J Am Coll Cardiol 2020;75:1281-95.

103. Gori M, Senni M, Gupta DK, et al. Association between renal function and cardiovascular structure and function in heart failure with preserved ejection fraction. Eur Heart J 2014;35:3442-51.

104. Collier P, Watson CJ, Voon V, et al. Can emerging biomarkers of myocardial remodelling identify asymptomatic hypertensive patients at risk for diastolic dysfunction and diastolic heart failure? Eur J Heart Fail 2011;13:1087-95.

105. Tromp J, Westenbrink BD, Ouwerkerk W, et al. Identifying pathophysiological mechanisms in heart failure with reduced versus preserved ejection fraction. J Am Coll Cardiol 2018;72:1081-90.

106. Cohen JB, Schrauben SJ, Zhao L, et al. Clinical phenogroups in heart failure with preserved ejection fraction: detailed phenotypes, prognosis, and response to spironolactone. JACC Heart Fail 2020;8:172-84.

107. Schiattarella GG, Altamirano F, Tong D, et al. Nitrosative stress drives heart failure with preserved ejection fraction. Nature 2019;568:351-6.

108. Van Tassell BW, Arena R, Biondi-Zoccai G, et al. Effects of interleukin-1 blockade with anakinra on aerobic exercise capacity in patients with heart failure and preserved ejection fraction (from the D-HART pilot study). Am J Cardiol 2014;113:321-7.

109.Van Tassell BW, Trankle CR, Canada JM, et al. IL-1 blockade in patients with heart failure with preserved ejection fraction. Circ Heart Fail 2018;11:e005036.

110.Blanton RM, Carrillo-Salinas FJ, Alcaide P. T-cell recruitment to the heart: friendly guests or unwelcome visitors? Am J Physiol Heart Circ Physiol 2019;317:H124-40.

111.DeBerge M, Shah SJ, Wilsbacher L, et al. Macrophages in heart failure with reduced versus preserved ejection fraction. Trends Mol Med 2019;25:328-40.

112.Hulsmans M, Sager HB, Roh JD, et al. Cardiac macrophages promote diastolic dysfunction. J Exp Med 2018;215:423-40.

113.van Heerebeek L, Hamdani N, Falcao-Pires I, et al. Low myocardial protein kinase $\mathrm{G}$ activity in heart failure with preserved ejection fraction. Circulation 2012;126:830-9.

114. Vergaro G, Aimo A, Prontera C, et al. Sympathetic and renin-angiotensin-aldosterone system activation in heart failure with preserved, mid-range and reduced ejection fraction. Int J Cardiol 2019;296:91-7.

115. Benedict CR, Johnstone DE, Weiner DH, et al. Relation of neurohumoral activation to clinical variables and degree of ventricular dysfunction: a report from the Registry of Studies of Left Ventricular Dysfunction. SOLVD Investigators. J Am Coll Cardiol 1994;23:1410-20.

116.Seravalle G, Quarti-Trevano F, Dell'Oro R, et al. Sympathetic and baroreflex alterations in congestive heart failure with preserved, midrange and reduced ejection fraction. J Hypertens 2019;37:443-8.

117. Catena C, Verheyen N, Pilz S, et al. Plasma aldosterone and left ventricular diastolic function in treatment-naive patients with hypertension: tissue-Doppler imaging study. Hypertension 2015;65:1231-7.

118. Kuno T, Ueyama H, Fujisaki T, et al. Meta-analysis evaluating the effects of renin-angiotensin-aldosterone system blockade on outcomes of heart failure with preserved ejection fraction. Am J Cardiol 2020;125:1187-93.

119.Paulus WJ, van Ballegoij JJ. Treatment of heart failure with normal ejection fraction: an inconvenient truth! J Am Coll Cardiol 2010;55:526-37.

120. Fudim M, Kelly JP, Brophy TJ, et al. Trends in treatment for patients hospitalized with heart failure with preserved ejection fraction before and after treatment of preserved cardiac function heart failure with an aldosterone antagonist (TOPCAT). Am J Cardiol 2020;125:1655-60.

121. Upadhya B, Kitzman DW. Beta-blockers for primary therapy of heart failure with preserved ejection fraction: an idea whose time has gone? J Card Fail 2020;26:283-4.

122.Palau P, Seller J, Dominguez E, et al. Beta-blockers withdrawal in patients with heart failure with preserved ejection fraction and chronotropic incompetence: Effect on functional capacity rationale and study design of a prospective, randomized, controlled trial (The PreserveHR trial). Clin Cardiol 2020;43:423-9.

123. Bisping E, Wakula P, Poteser M, et al. Targeting cardiac hypertrophy: towards a causal heart failure therapy. J Cardiovasc Pharmacol 2014;64:293-305.

124. Blanton RM. cGMP signaling and modulation in heart failure. J Cardiovasc Pharmacol 2020;75:385-98.

125. Gorter TM, Hoendermis ES, van Veldhuisen DJ, et al. Right ventricular dysfunction in heart failure with preserved ejection fraction: a systematic review and meta- 
analysis. Eur J Heart Fail 2016;18:1472-87.

126. Platt MJ, Huber JS, Romanova N, et al. Pathophysiological mapping of experimental heart failure: left and right ventricular remodeling in transverse aortic constriction is temporally, kinetically and structurally distinct. Front Physiol 2018;9:472.

127. Cenkerova K, Dubrava J, Pokorna V, et al. Right ventricular systolic dysfunction and its prognostic value in heart failure with preserved ejection fraction. Acta Cardiol 2015;70:387-93

128. Puwanant S, Priester TC, Mookadam F, et al. Right ventricular function in patients with preserved and reduced ejection fraction heart failure. Eur J Echocardiogr 2009; 10:733-7.

129. Damy T, Kallvikbacka-Bennett A, Goode K, et al. Prevalence of, associations with, and prognostic value of tricuspid annular plane systolic excursion (TAPSE) among out-patients referred for the evaluation of heart failure. J Card Fail 2012;18:216-25.

130. Morris DA, Gailani M, Vaz Perez A, et al. Right ventricular myocardial systolic and diastolic dysfunction in heart failure with normal left ventricular ejection fraction. J Am Soc Echocardiogr 2011;24:886-97.

131. Mohammed SF, Hussain I, AbouEzzeddine OF, et al. Right ventricular function in heart failure with preserved ejection fraction: a community-based study. Circulation 2014;130:2310-20.

132. Ghio S, Guazzi M, Scardovi AB, et al. Different correlates but similar prognostic implications for right ventricular dysfunction in heart failure patients with reduced or preserved ejection fraction. Eur J Heart Fail 2017;19:873-9.

133. Melenovsky V, Hwang SJ, Lin G, et al. Right heart dysfunction in heart failure with preserved ejection fraction. Eur Heart J 2014;35:3452-62.

134. Breitling S, Ravindran K, Goldenberg NM, et al. The pathophysiology of pulmonary hypertension in left heart disease. Am J Physiol Lung Cell Mol Physiol 2015;309:L924-41.

135. Naeije R, Vachiery JL, Yerly P, et al. The transpulmonary pressure gradient for the diagnosis of pulmonary vascular disease. Eur Respir J 2013;41:217-23.

136.Pieske B, Tschope C, de Boer RA, et al. How to diagnose heart failure with preserved ejection fraction: the HFAPEFF diagnostic algorithm: a consensus recommendation from the Heart Failure Association (HFA) of the European Society of Cardiology (ESC). Eur Heart J 2019;40:3297-317.
137. Rosenkranz S, Lang IM, Blindt R, et al. Pulmonary hypertension associated with left heart disease: updated recommendations of the Cologne Consensus Conference 2018. Int J Cardiol 2018;272S:53-62.

138. Gorter TM, van Veldhuisen DJ, Bauersachs J, et al. Right heart dysfunction and failure in heart failure with preserved ejection fraction: mechanisms and management. Position statement on behalf of the Heart Failure Association of the European Society of Cardiology. Eur J Heart Fail 2018;20:16-37.

139. Douschan P, Kovacs G, Avian A, et al. Mild elevation of pulmonary arterial pressure as a predictor of mortality. Am J Respir Crit Care Med 2018;197:509-16.

140. Maron BA, Hess E, Maddox TM, et al. Association of borderline pulmonary hypertension with mortality and hospitalization in a large patient cohort: insights from the Veterans Affairs Clinical Assessment, Reporting, and Tracking Program. Circulation 2016;133:1240-8.

141. Condon DF, Nickel NP, Anderson R, et al. The 6th World Symposium on Pulmonary Hypertension: what's old is new. F1000Res 2019;8:F1000 Faculty Rev-888.

142. Thenappan T, Shah SJ, Gomberg-Maitland M, et al. Clinical characteristics of pulmonary hypertension in patients with heart failure and preserved ejection fraction. Circ Heart Fail 2011;4:257-65.

143. Vachiéry JL, Adir Y, Barberà JA, et al. Pulmonary hypertension due to left heart diseases. J Am Coll Cardiol 2013;62:D100-8.

144. Charalampopoulos A, Lewis R, Hickey P, et al. Pathophysiology and diagnosis of pulmonary hypertension due to left heart disease. Front Med (Lausanne) 2018;5:174.

145. Gerges M, Gerges C, Pistritto AM, et al. Pulmonary hypertension in heart failure. epidemiology, right ventricular function, and survival. Am J Respir Crit Care Med 2015;192:1234-46.

146. Lam CS, Roger VL, Rodeheffer RJ, et al. Pulmonary hypertension in heart failure with preserved ejection fraction: a community-based study. J Am Coll Cardiol 2009;53:1119-26.

147. Borlaug BA. The pathophysiology of heart failure with preserved ejection fraction. Nat Rev Cardiol 2014;11:507-15.

148. Kuebler WM. Effects of pressure and flow on the pulmonary endothelium. In: Voelkel NF, Rounds S. editors. The pulmonary endothelium: function in health and disease. Wiley: Hoboken, 2009.

149.Kerem A, Yin J, Kaestle SM, et al. Lung endothelial 
dysfunction in congestive heart failure: role of impaired $\mathrm{Ca} 2+$ signaling and cytoskeletal reorganization. Circ Res 2010;106:1103-16.

150. Hunt JM, Bethea B, Liu X, et al. Pulmonary veins in the normal lung and pulmonary hypertension due to left heart disease. Am J Physiol Lung Cell Mol Physiol 2013;305:L725-36.

151.Sunagawa K, Maughan WL, Burkhoff D, et al. Left ventricular interaction with arterial load studied in isolated canine ventricle. Am J Physiol 1983;245:H773-80.

152.Hsu S. Coupling Right ventricular-pulmonary arterial research to the pulmonary hypertension patient bedside. Circ Heart Fail 2019;12:e005715.

153. Obokata M, Reddy YNV, Melenovsky V, et al. Deterioration in right ventricular structure and function over time in patients with heart failure and preserved ejection fraction. Eur Heart J 2019;40:689-97.

154.Spinner EM, Shannon P, Buice D, et al. In vitro characterization of the mechanisms responsible for functional tricuspid regurgitation. Circulation 2011;124:920-9.

155.Foschi M, Di Mauro M, Tancredi F, et al. The dark side of the moon: the right ventricle. J Cardiovasc Dev Dis 2017;4:18.

156. Rana BS, Robinson S, Francis R, et al. Tricuspid regurgitation and the right ventricle in risk stratification and timing of intervention. Echo Res Pract 2019;6:R25-39.

157. Dittrich HC, Chow LC, Nicod PH. Early improvement in left ventricular diastolic function after relief of chronic right ventricular pressure overload. Circulation 1989;80:823-30.

158. Friedberg MK, Redington AN. Right versus left ventricular failure: differences, similarities, and interactions. Circulation 2014;129:1033-44.

159.Sun M, Ishii R, Okumura K, et al. Experimental right ventricular hypertension induces regional beta1-integrinmediated transduction of hypertrophic and profibrotic right and left ventricular signaling. J Am Heart Assoc 2018;7:e007928.

160. Chen S, Zhang Y, Lighthouse JK, et al. A novel role of cyclic nucleotide phosphodiesterase $10 \mathrm{~A}$ in pathological cardiac remodeling and dysfunction. Circulation 2020;141:217-33.

161.Xue M, Li T, Wang Y, et al. Empagliflozin prevents cardiomyopathy via sGC-cGMP-PKG pathway in type 2 diabetes mice. Clin Sci (Lond) 2019;133:1705-20.

162.Lewis GA, Schelbert EB, Naish JH, et al. Pirfenidone in heart failure with preserved ejection fraction-rationale and design of the PIROUETTE trial. Cardiovasc Drugs Ther 2019;33:461-70.

163. Ibrahim NE, Januzzi JL Jr. Established and emerging roles of biomarkers in heart failure. Circ Res 2018;123:614-29.

164. Tromp J, Khan MA, Klip IT, et al. Biomarker profiles in heart failure patients with preserved and reduced ejection fraction. J Am Heart Assoc 2017;6:e003989.
Cite this article as: Heinzel FR, Hegemann N, Hohendanner F, Primessnig U, Grune J, Blaschke F, de Boer RA, Pieske B, Schiattarella GG, Kuebler WM. Left ventricular dysfunction in heart failure with preserved ejection fraction-molecular mechanisms and impact on right ventricular function. Cardiovasc Diagn Ther 2020;10(5):1541-1560. doi: 10.21037/cdt$20-477$ 